\title{
Article
}

\section{Identification of Phytaspase Interactors via the Proximity-Dependent Biotin-Based Identification Approach}

\author{
Anastasia D. Teplova ${ }^{1}$, Marina V. Serebryakova ${ }^{2}{ }^{(D}$, Raisa A. Galiullina ${ }^{2}$, Nina V. Chichkova ${ }^{2}$ \\ and Andrey B. Vartapetian 2,*(D) \\ 1 Faculty of Bioengineering and Bioinformatics, Lomonosov Moscow State University, Moscow 119991, Russia; \\ anastasia_teplova@mail.ru \\ 2 Belozersky Institute of Physico-Chemical Biology, Lomonosov Moscow State University, \\ Moscow 119991, Russia; mserebr@mail.ru (M.V.S.); raisa-galiullina@rambler.ru (R.A.G.); \\ chic@genebee.msu.ru (N.V.C.) \\ * Correspondence: varta@genebee.msu.ru
}

check for updates

Citation: Teplova, A.D.;

Serebryakova, M.V.; Galiullina, R.A.; Chichkova, N.V.; Vartapetian, A.B. Identification of Phytaspase Interactors via the Proximity-Dependent Biotin-Based Identification Approach. Int. J. Mol. Sci. 2021, 22, 13123. https://doi.org/ $10.3390 /$ ijms 222313123

Academic Editor: Yasuhito Sakuraba

Received: 5 November 2021

Accepted: 2 December 2021

Published: 4 December 2021

Publisher's Note: MDPI stays neutral with regard to jurisdictional claims in published maps and institutional affiliations.

Copyright: (c) 2021 by the authors Licensee MDPI, Basel, Switzerland. This article is an open access article distributed under the terms and conditions of the Creative Commons Attribution (CC BY) license (https:// creativecommons.org/licenses/by/ $4.0 /)$.

\begin{abstract}
Proteolytic enzymes are instrumental in various aspects of plant development, including senescence. This may be due not only to their digestive activity, which enables protein utilization, but also to fulfilling regulatory functions. Indeed, for the largest family of plant serine proteases, subtilisin-like proteases (subtilases), several members of which have been implicated in leaf and plant senescence, both non-specific proteolysis and regulatory protein processing have been documented. Here, we strived to identify the protein partners of phytaspase, a plant subtilase involved in stress-induced programmed cell death that possesses a characteristic aspartate-specific hydrolytic activity and unusual localization dynamics. A proximity-dependent biotin identification approach in Nicotiana benthamiana leaves producing phytaspase fused to a non-specific biotin ligase TurboID was employed. Although the TurboID moiety appeared to be unstable in the apoplast environment, several intracellular candidate protein interactors of phytaspase were identified. These were mainly, though not exclusively, represented by soluble residents of the endoplasmic reticulum, namely endoplasmin, BiP, and calreticulin-3. For calreticultin-3, whose gene is characterized by an enhanced expression in senescing leaves, direct interaction with phytaspase was confirmed in an in vitro binding assay using purified proteins. In addition, an apparent alteration of post-translational modification of calreticultin-3 in phytaspase-overproducing plant cells was observed.
\end{abstract}

Keywords: plant protease; phytaspase; protein interactor; BioID; endoplasmic reticulum; calreticulin-3; senescence

\section{Introduction}

Subtilisin-like proteases represent the largest family of plant serine proteases that are involved in various aspects of plant life, including growth, development, senescence, programmed cell death, and stress responses [1]. Subtilases comprise over 50 members in different plant organisms, for example, 56 are found in Arabidopsis thaliana [2]. In relation to senescence, in $A$. thaliana, the mRNA level and proteolytic activity of subtilisin-like protease AtSBT1.4, a senescence-associated protease (SASP), was reported to increase in the leaves of senescing during the vegetative or reproductive phase [3]. Induction of subtilisinlike proteases was also observed in wheat leaves under dark- and $\mathrm{N}$-starvation-induced senescence and in naturally senescing plants $[4,5]$. Within the subtilase family of proteolytic enzymes, phytaspases are distinguished by their strict substrate cleavage specificity after an aspartate residue, preceded by a characteristic (though degenerate) tripeptide amino acid motif [6-9]. Due to this type of recognition, similar to that of animal apoptotic proteases (caspases), phytaspases are processive (that is, they introduce single breaks into a limited number of protein substrates), rather than digestive, proteolytic enzymes. In 
continuation of the analogy with caspases, phytaspases were shown to be instrumental in the accomplishment of plant cell death induced by biotic and abiotic stresses [6,10-13].

Another intriguing feature of phytaspases is their dynamic localization. Phytaspase precursors are equipped with a signal peptide that guides the proenzyme to the endoplasmic reticulum with further secretion from the plant cell. In the course of this anterograde transport, phytaspase precursor becomes autocatalytically and constitutively processed, and thus activated with concomitant release and accumulation of the mature enzyme in the apoplast [6]. However, this is not the end of phytaspase transportation. Upon induction of cell death by biotic and abiotic stresses, phytaspases utilize clathrin-mediated endocytosis for retrograde transport into the plant cells [14,15].

To elucidate the molecular mechanisms of phytaspase localization dynamics, we employed an in planta proximity-dependent biotin identification (BioID) approach [16] to identify phytaspase interactors in Nicotiana benthamiana leaves. In this study, phytaspase interaction with calreticulin-3, the only plant calreticulin isoform that is up-regulated in senescing leaves [17], was uncovered in vivo and confirmed in vitro.

\section{Results}

\subsection{Setup of the in Planta Biotinylation Assay}

To obtain a Nicotiana tabacum phytaspase (NtPhyt) derivative competent for the BioID assay, we fused the non-specific biotin ligase TurboID [18] to the NtPhyt precursor. The choice for the TurboID version of biotin ligase was due to its efficient performance in plant cells relative to the previous variants of promiscuous biotin ligases [19-21]. As the NtPhyt precursor is subjected to $\mathrm{N}$-terminal processing (i.e., cleavage of the signal peptide and autocatalytic detachment of the N-terminal prodomain [6]), we fused TurboID to the carboxy terminus of the NtPhyt precursor to generate NtPhyt-TurboID $\sim 120 \mathrm{kDa}$ protein (Figure 1A).
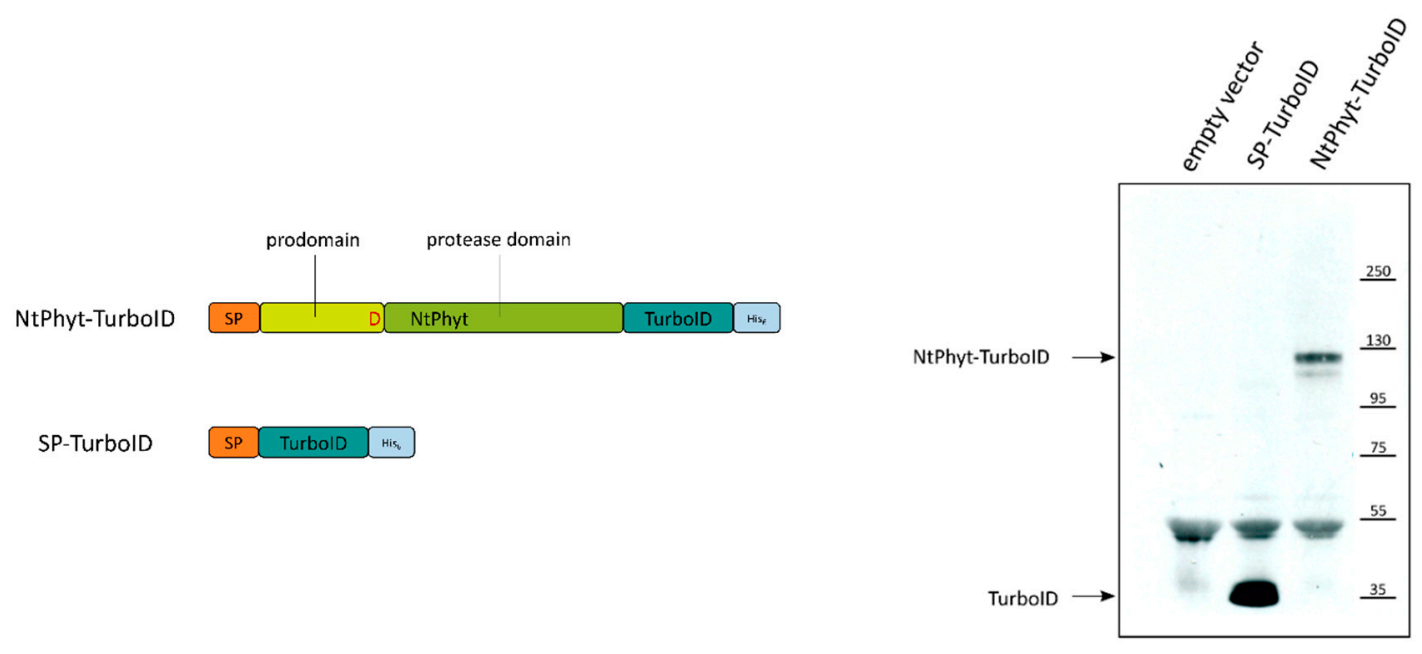

(a)

(b)

Figure 1. Nicotiana tabacum phytaspase (NtPhyt)-TurboID and signal peptide (SP)-TurboID proteins utilized for the identification of phytaspase interactors. (a) Schematic representation of recombinant proteins. Non-specific biotin ligase TurboID fused to SP derived from NtPhyt served as a control for in planta biotinylation accomplished by NtPhyt-TurboID protein. Both proteins bear His tags to permit their detection using Western blotting analysis. (b) Transient production of NtPhyt-TurboID and SP-TurboID in N. benthamiana leaves. Protein extracts from NtPhyt-TurboID-producing, SP-TurboIDproducing and control (vector) leaves were fractionated by sodium dodecyl sulphate (SDS) gel-electrophoresis and analysed by Western blotting with HisProbe detection. Positions of molecular weight protein markers are indicated on the right.

A free TurboID derivative was also constructed as a control for non-specific TurboIDmediated biotinylation. To mimic NtPhyt anterograde transportation, TurboID was equipped 
with an N-terminal signal peptide derived from NtPhyt to generate SP-TurboID $\sim 35 \mathrm{kDa}$ protein (Figure 1A). In addition, both proteins were supplied with hexahistidine tags at their C-termini to allow visualization of their synthesis and accumulation in plant cells.

Synthesis of either NtPhyt-TurboID or SP-TurboID in N. benthamiana was achieved by leaf infiltration with agrobacteria carrying a plasmid with the respective gene under the control of a strong constitutive $35 \mathrm{~S}$ promoter. Figure $1 \mathrm{~B}$ demonstrates that the production of both recombinant proteins was readily detectable by Western blot analysis of extracts of infiltrated leaves.

\subsection{Stability of NtPhyt-TurboID and SP-TurboID Proteins inside and outside the Plant Cells}

To assess the stability of recombinant proteins, both within the cell and in the apoplast, apoplastic washes were obtained from leaf tissues producing each protein. The amounts of recombinant proteins in the apoplast were compared with those in the residual leaf tissue (after separation of the apoplast) by Western blotting. As shown in Figure 2A, both proteins were clearly detectable within the intracellular fraction and thus turned out to be quite stable. However, in the apoplastic fraction, only small amounts of TurboID (derived from SP-TurboID) were visible, whereas NtPhyt-TurboID was undetectable.

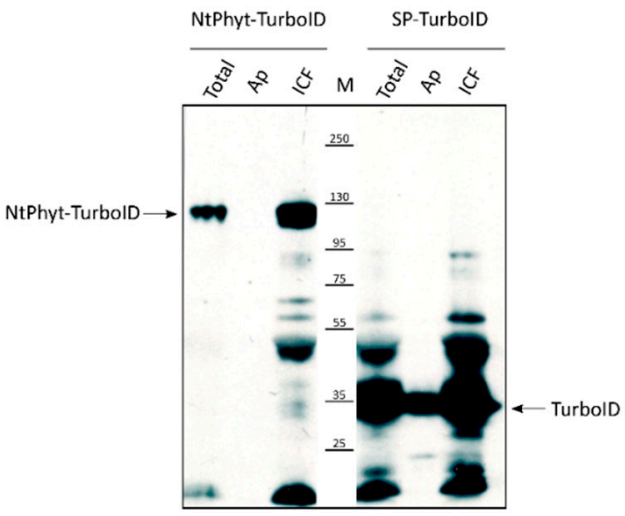

(a)

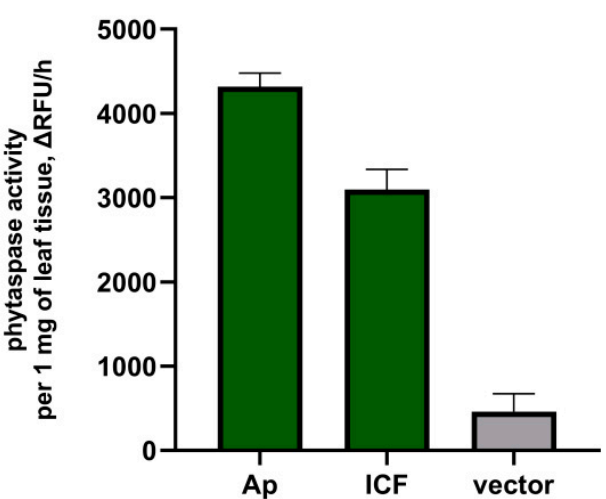

(b)

Figure 2. Evaluation of the stability of NtPhyt-TurboID and SP-TurboID proteins in the apoplast and inside the plant cells. (a) For NtPhyt-TurboID- and SP-TurboID-producing leaves, proteins in the apoplastic washes (Ap) and intracellular fractions (ICFs) were analysed by Western blotting with HisProbe detection. 'Total' represents leaf extracts without fractionation. Equal amounts of leaf tissues (5 mg) were taken for protein analyses, and equivalent $15 \mu \mathrm{L}$ aliquots of the subcellular fractions were loaded on the gel. M, molecular weights of protein markers. Arrows indicate positions of the recombinant proteins. (b) Measurement of phytaspase proteolytic activity in the extracellular (Ap) and intracellular (ICF) fractions obtained from NtPhyt-TurboID-producing leaves. 'Vector' total protein sample from leaves infiltrated with agrobacteria carrying the empty vector. Ac-VEID-AFC $(20 \mu \mathrm{M})$ was used as the phytaspase substrate for quantitative assessment of phytaspase proteolytic activity. Relative rates of hydrolysis were determined as an increase of relative fluorescence units per hour (deltaRFU/h). Enzymatic activities were normalized by the weight of leaf tissues taken for analysis. Data represent the mean of three independent experiments \pm standard deviation (SD).

From our previous studies, we know that the signal peptide of the NtPhyt precursor guides very efficient secretion of NtPhyt itself and of heterologous proteins [6,15]. Therefore, we suspected that the TurboID moiety, once secreted, is unstable in the apoplast. To verify whether NtPhyt synthesized as a NtPhyt-TurboID fusion protein is competent for secretion, we compared the levels of NtPhyt proteolytic activity in the apoplastic and intracellular fractions using Ac-VEID-AFC, the preferred fluorogenic peptide substrate of $N t$ Phyt [6]. Figure 2B shows that the level of NtPhyt activity in the apoplast of NtPhytTurboID-producing leaves markedly exceeded the level of endogenous phytaspase activity, 
indicating that secretion of the enzyme was not impaired. Together with the observed stability of the NtPhyt-TurboID protein within the cell, this finding is consistent with the degradation of the TurboID moiety upon secretion of the protein into the apoplast.

\subsection{Candidate Phytaspase Interactors Revealed by BioID Approach}

To identify potential NtPhyt interactors, detached N. benthamiana leaves producing either NtPhyt-TurboID or SP-TurboID were infiltrated with $200 \mu \mathrm{M}$ biotin solution containing $500 \mu \mathrm{M}$ ATP to support TurboID-mediated protein biotinylation and incubated for $5 \mathrm{~h}$. Inspection of protein extracts obtained from these leaves using Western blot analysis with streptavidin-horseradish peroxidase (HRP) visualization revealed the presence of multiple biotinylated proteins in total leaf extracts of the control (SP-TurboID-producing) and NtPhyt-TurboID-containing samples (Figure 3) and in the intracellular fractions, but not in the respective apoplastic fractions (Figure S1).

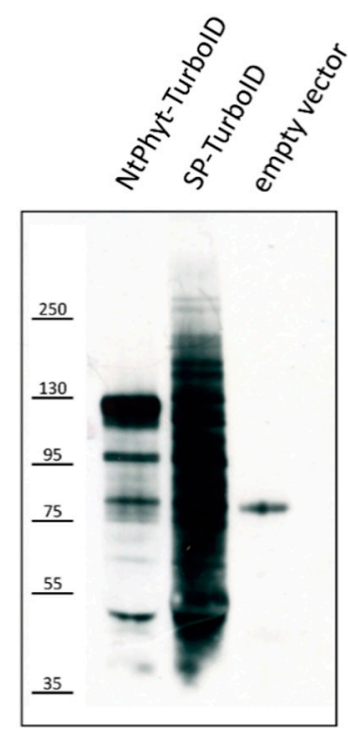

Figure 3. Protein biotinylation in N. benthamiana leaves producing either NtPhyt-TurboID or SPTurboID proteins. Western blotting analysis with streptavidin-horseradish peroxidase (HRP) detection of total proteins biotinylated in N. benthamiana leaves producing either NtPhyt-TurboID or SP-TurboID proteins. 'Vector,' total protein sample from leaves infiltrated with agrobacteria carrying the empty vector. Equal amounts of leaf tissues $(5 \mathrm{mg})$ were taken for analysis, and equivalent $15 \mu \mathrm{L}$ aliquots of the subcellular fractions were loaded. Positions of molecular weight protein markers are indicated on the left.

In order to simplify patterns of biotinylated proteins in the intracellular fractions, intracellular protein samples were further fractionated to obtain soluble proteins (that is, extractable in the absence of detergents) and 'membrane' proteins (extractable in the presence of $0.5 \%$ dodecyl maltoside detergent). After subsequent protein fractionation using ammonium sulphate precipitation and affinity chromatography using magnetic streptavidin beads, biotinylated proteins from each fraction were analysed by Western blotting with streptavidin-HRP detection. The difference between NtPhyt-TurboID-containing and control samples was more clearly observed for the detergent-soluble fraction of proteins, as shown in Figure 4A. A number of protein bands that differed in the NtPhyt-TurboID and control SP-TurboID samples were observed. To identify candidate protein interactors, the same protein samples were compared by gel electrophoresis and Coomassie staining. The obtained patterns of stained proteins (Figure 4B) were similar to those visualized by streptavidin-HRP detection (Figure 4A), thus confirming the successful isolation of biotinylated proteins. Proteins from bands marked with numbers in Figure $4 \mathrm{~B}$ were subjected to trypsin hydrolysis and mass spectrometry (MS) identification. 


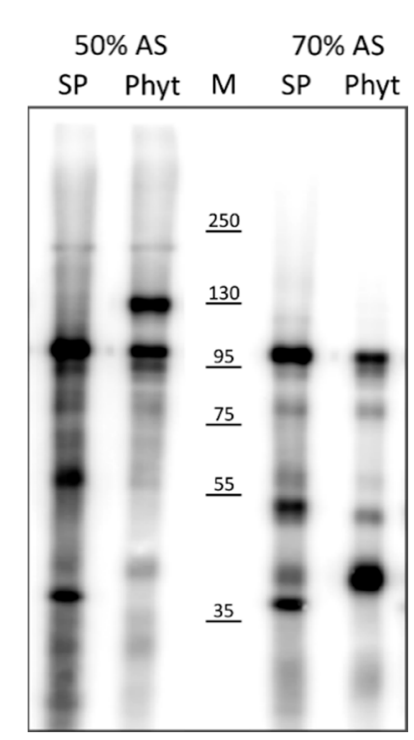

(a)

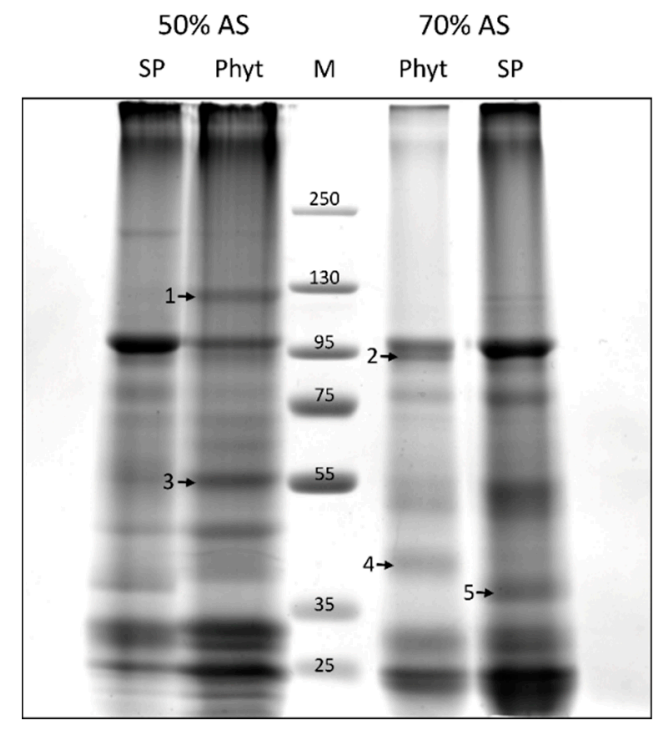

(b)

Figure 4. Visualizing the candidate protein interactors of NtPhyt. Proteins biotinylated in NtPhytTurboID- or SP-TurboID-producing leaves during the incubation of leaf samples with $200 \mu \mathrm{M}$ biotin for $5 \mathrm{~h}$ (Phyt and SP, respectively) were isolated from the respective intracellular fractions (obtained from equal $5 \mathrm{~g}$ amounts of leaf tissues) by extraction in the presence of $0.5 \%$ dodecyl maltoside. Upon ammonium sulphate (AS) fractionation, biotinylated proteins precipitated with $50 \%$ AS and $70 \%$ AS were separated by affinity chromatography on streptavidin magnetic beads and analysed by SDS gel electrophoresis. (a) On-blot detection of biotinylated proteins using streptavidin-HRP. One \% of the affinity purified protein samples in $4 \mu \mathrm{L}$ aliquots were loaded. (b) The rest of the samples, after acetone precipitation, were dissolved in $45 \mu \mathrm{L}$ of SDS-containing buffer and analysed by SDS gel electrophoresis. Coomassie blue staining of the $6-16 \%$ gradient polyacrylamide gel. Arrows with numbers indicate the bands chosen for protein identification by mass spectrometry (MS) analysis. M, molecular weights of the protein markers.

From these analyses, we learned that band 1 ( 120 kDa protein), which is present in the NtPhyt-Turbo sample only, corresponds to the recombinant protein NtPhyt-TurboID itself. Band 5 ( $\sim 37 \mathrm{kDa}$ protein) present in the control sample only corresponds to TurboID. These identifications were not surprising, as promiscuous biotin ligases are known to biotinylate themselves [18]. Band 2 ( $\sim 95 \mathrm{kDa}$ protein) present in the NtPhyt-TurboID sample corresponds to the endoplasmin homolog, a soluble resident of the endoplasmic reticulum. MS analysis revealed the presence of endoplasmin in the same position of the control (SP-TurboID) lane as well, although this was not evident from the protein patterns in Figure 4B. Bands $3(\sim 56 \mathrm{kDa}$ protein) and $4(\sim 40 \mathrm{kDa}$ protein) observed predominantly in the phytaspase-expressing sample were identified as ribulose-1,5-bisphosphate carboxylase/oxygenase large subunit and an uncharacterized protein LOC109229657, respectively. Analysis of the amino acid sequence of this latter protein revealed the presence of a signal peptide and a saposin B-type domain in its structure. Saposin B-type domains in known animal and plant membrane-interacting proteins have been reported to destabilize lipid membranes, with or without membrane permeabilization [22].

\subsection{Prolonged Incubation of Leaves with Biotin Revealed Additional Potential Phytaspase Interactors}

To increase the sensitivity of the assay, N. benthamiana leaves producing either NtPhytTurboID or SP-TurboID proteins were incubated with biotin for $16 \mathrm{~h}$. Fractionation of leaf extracts was performed, as described in the previous section. Representative patterns of biotinylated proteins from the detergent-soluble fraction isolated by affinity chromatography from both samples and stained with Coomassie are shown in Figure 5. Apart from MS re-identification of NtPhyt-TurboID Figure 5, band 1) and the endoplasmin homolog 
(Figure 5, band 2), two novel candidate interactors were identified. Band 3 in the NtPhytTurboID sample corresponds to $\mathrm{BiP}$ (immunoglobulin binding protein), an endoplasmic reticulum luminal binding protein belonging to the Hsp70 protein family. Although a faint band with similar electrophoretic mobility was observed in the control sample (band 3c), MS analysis failed to identify BiP as a TurboID interactor. Thus, BiP appears to specifically interact with NtPhyt.

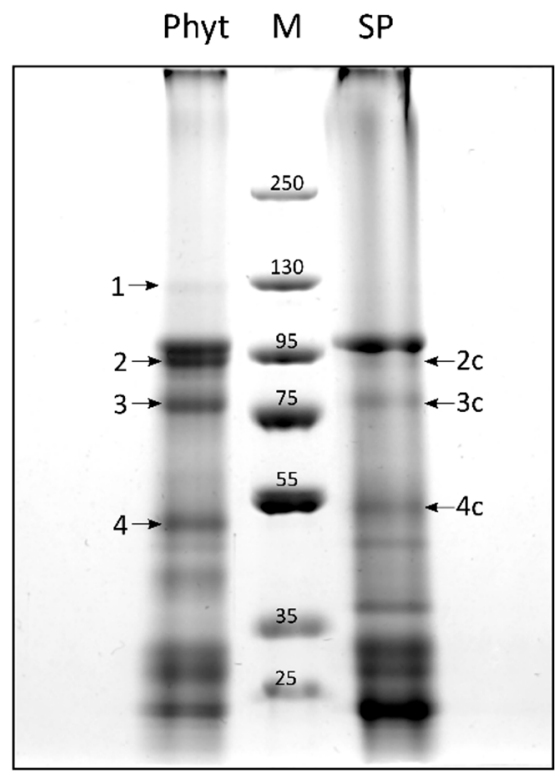

Figure 5. Longer incubation of leaf samples with biotin reveals new candidate phytaspase interactors. After prolonged $(16 \mathrm{~h})$ incubation of leaf samples with $200 \mu \mathrm{M}$ biotin, proteins were extracted from the intracellular fractions of NtPhyt-TurboID- or SP-TurboID-producing leaves (Phyt and SP, respectively; obtained from equal $5 \mathrm{~g}$ amounts of leaf material) in the presence of $0.5 \%$ dodecyl maltoside. Biotinylated proteins that precipitated within the $50-70 \%$ interval of ammonium sulphate saturation were further affinity-purified using streptavidin magnetic beads. The eluted protein samples were concentrated by acetone precipitation, dissolved in $45 \mu \mathrm{L}$ of SDS-containing buffer and analysed by SDS electrophoresis in a $6-16 \%$ gradient polyacrylamide gel. Proteins were visualized after Coomassie Blue staining. M, molecular weights of the protein markers. Arrows with numbers indicate the bands chosen for subsequent protein identification.

Another candidate protein (band 4 in Figure 5, 52kDa) was identified as calreticulin3 , an endoplasmic reticulum lumen protein [23] known to function as a chaperone and a calcium-binding protein. Calreticulin-3 was identified in both the NtPhyt-TurboID and control samples (bands 4 and 4c, respectively), although its electrophoretic mobility appeared to be slightly slower in the control. This might be indicative of alterations in post-translational modification in phytaspase-overproducing leaves.

Table 1 summarizes our findings in identifying possible NtPhyt interactors using the BioID approach. The majority of proteins identified (endoplasmin, BiP, and calreticulin-3) represent known residents of the endoplasmic reticulum lumen that function as chaperones [23]. Of these, only BiP appears to represent a "true" candidate phytaspase interactor, as it was not detected in the control (SP-TurboID-producing) sample. However, for calreticulin-3, the impact of phytaspase may also be expected, as this protein displays slightly altered mobility in the NtPhyt-TurboID-producing samples. Another example of a specific interactor absent in control probes is an uncharacterized saposin B-type domaincontaining protein. As for the Rubisco subunit, a protein of chloroplast origin, we do not yet know whether its identification was due to the enormous abundance of this protein in plant cells, as the presence of the corresponding biotinylated protein was not evident in Figure 4a. 
Table 1. Biotinylated proteins-candidate phytaspase interactors identified in this study.

\begin{tabular}{|c|c|c|c|c|}
\hline Name. & Identification & $\mathrm{Mr}$ & Fraction & Presence in the Control \\
\hline Endoplasmin & XP_019239585.1 & $95 \mathrm{kDa}$ & detergent soluble & Yes \\
\hline $\mathrm{BiP}$ & XP_016484998.1 & $78 \mathrm{kDa}$ & $\begin{array}{l}\text { detergent soluble } \\
\text { water soluble }\end{array}$ & No \\
\hline Rubisco (large subunit) & NP_054507.1 & $56 \mathrm{kDa}$ & detergent soluble & No \\
\hline Calreticulin-3 & XP_016452363.1 & $52 \mathrm{kDa}$ & detergent soluble & $\begin{array}{c}\text { Yes, } \\
\text { lower mobility } \\
\text { in the control }\end{array}$ \\
\hline $\begin{array}{l}\text { Saposin B type domain } \\
\text { containing protein }\end{array}$ & $\begin{array}{c}\text { LOC109229657 } \\
\text { XP_019250726.1 }\end{array}$ & $40 \mathrm{kDa}$ & detergent soluble & No \\
\hline
\end{tabular}

For proteins listed in Table 1, sequence coverage with the MS-identified peptides is given in Figure S2.

\subsection{Calreticulin-3 Interacts with NtPhytaspase In Vitro}

To verify the results of the in planta BioID approach and to clarify whether calreticulin3 might represent a true phytaspase partner, the possibility of direct interaction between purified N. tabacum calreticulin-3 and NtPhyt was assessed. Recombinant N. tabacum calreticulin-3, in which the signal peptide was substituted with a His6 tag (His-CRT3), was overproduced in E. coli cells and immobilized on Ni-NTA agarose. Untagged NtPhyt was overproduced and affinity-purified using a specific and reversible NtPhyt inhibitor from $N$. benthamiana leaves [6]. Upon incubation with immobilized calreticulin-3, the amount of NtPhyt proteolytic activity in the flow-through (unbound enzyme) and in the eluate from the column with EDTA-containing buffer (releasing the potential His-CRT3-NtPhyt complex) was quantitatively determined. Ni-NTA agarose resin preincubated with an equivalent amount of lysate of vector-only transformed $E$. coli cells served as a control. As shown in Figure 6A, phytaspase activity was reduced in the flow-through from the calreticulin-3-containing resin, relative to the control sample, while it was enhanced in the eluate (Figure 6B), thus providing evidence for direct calreticulin-3-phytaspase interaction.

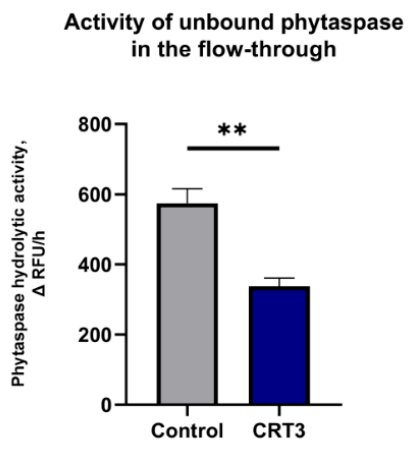

(a)

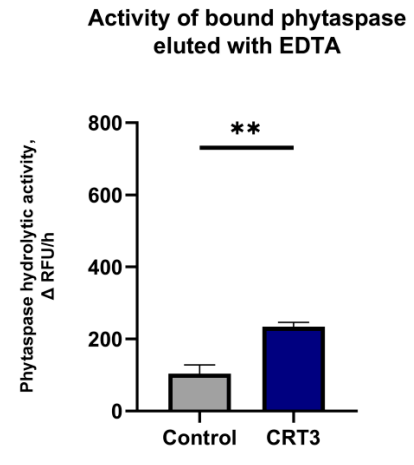

(b)

Figure 6. Evidence for direct in vitro interaction of NtPhyt with calreticulin-3. Recombinant $N$. tabacum calreticulin-3 bearing an N terminal His6 tag (His-CRT3) was produced in Escherichia coli cells and immobilized on Ni-nitrilotriacetic acid (Ni-NTA) agarose (CRT3, approx. $0.5 \mu$ g protein per binding experiment). Ni-NTA agarose resin preincubated with an equivalent amount of lysed vector-only transformed E. coli cells was used as a control (control). Upon incubation of each resin with $N t$ Phyt for $1 \mathrm{~h}, N t$ Phyt proteolytic activity in the flow-through fractions (a) and in the eluates from the column with EDTA-containing buffer (b) was fluorometrically determined using $20 \mu \mathrm{M}$ Ac-VEID-AFC as a phytaspase substrate. Data represent the mean \pm SD of three independent experiments. Significant differences to the control are shown as ${ }^{* *} p<0.01$. The two-tailed $p$ value is 0.0010 for (a) and 0.0011 for (b) (unpaired $t$-test). 


\section{Discussion}

BioID is a powerful tool for the identification of protein interactors in living cells by means of proximity-dependent biotinylation, which was recently successfully applied in plant systems $[19-21,24,25]$. We used this approach to identify candidate protein partners of $N$. tabacum phytaspase, a subtilisin-like protease involved in the accomplishment of plant cell death induced by biotic and abiotic stresses $[6,10,12]$. As with the majority of plant subtilases, phytaspase is secreted into the apoplast of healthy plant tissues. However, a peculiarity of phytaspase is its retrograde transport from the apoplast back into plant cells that employs clathrin-mediated endocytosis and occurs upon the induction of programmed cell death $[14,15]$. By utilizing the BioID approach, we were hoping to identify proteins involved in phytaspase mobility.

In this study, we used a TurboID version of biotin ligase, as previous studies have shown that TurboID outperforms other non-specific biotin ligases for studies in plant cells [19-21]. For our search of NtPhyt interactors, a closely related N. benthamiana species was chosen as a host, as it is a widely used model plant, for which the BioID approach has been optimized [20]. However, we discovered a limitation of our scheme associated with an apparent instability of the TurboID moiety within the aggressive $N$. benthamiana apoplast environment. This might explain why we did not detect the extracellular partners of phytaspase. On the other hand, the absence or low abundance of stable phytaspaseinteracting proteins in the apoplast cannot be excluded.

Intracellular NtPhyt-TurboID was fairly stable, and this allowed us to identify a set of candidate protein interactors within the plant cell. Interestingly, three of these proteins (endoplasmin, BiP, and calreticulin-3) represent soluble residents of the lumen of the endoplasmic reticulum. They are known to perform chaperone and protein quality control functions for newly synthesized proteins targeted to the endoplasmic reticulum and serve as calcium ion stores (calreticulin) [23]. As the phytaspase precursor protein has to pass through the endoplasmic reticulum on the route of the enzyme from the plant cell, phytaspase contacts with the protein quality control system are intuitively understandable. However, it is not uncommon, both in plants and in animals, to find these endoplasmic reticulum "residents" at various additional locations, such as the plasma membrane, cell surface, nucleus, and cytoplasm, where these proteins were reported to perform important tasks [26-29]. Therefore, the possibility of phytaspase interaction with these proteins at locations other than the endoplasmic reticulum cannot be completely dismissed.

The specificity of phytaspase interaction with newly detected candidate proteins also deserves comment. Given that phytaspase is synthesized as an NtPhyt-TurboID fusion protein, who is involved in the interaction with the partner: phytaspase, TurboID, or both? Employment of the described SP-TurboID control construct was helpful in cases where the protein of interest was absent in the control track, such as BiP and saposin B-type domain-containing protein. However, in the case of endoplasmin and calreticulin-3, where the corresponding protein was identified in the control sample as well, the situation was less clear. The hint that phytaspase may be involved in the interaction with calreticulin-3 followed indirectly from a slightly altered electrophoretic mobility of the partner (possibly due to some post-translational modification) in the case of NtPhyt-TurboID production. Therefore, calreticulin- 3 was chosen as a target to verify the conclusions obtained using the BioID approach and to address the issue of specificity in this complicated case. Our in vitro assay with the purified Ntcalreticulin-3 and NtPhyt proteins supported the conclusion that these two proteins are capable of direct interaction. Calreticulin-3 is a member of calreticulin protein family consisting in plants of 3 members which have acquired specialized functions [17,30]. Plant calreticulin-3 has been reported to play important roles in maintaining plant innate immunity and in protecting plants against fungal and bacterial pathogens [31-35]. Of note, an enhanced expression of Arabidopsis thaliana CRT-3 gene in senescing leaves was reported, that contrasted markedly the expression patterns of the two other family members, which were much reduced [17]. As our BioID analysis identified calreticulin-3, but not the other family members, as a phytaspase partner, a functional 
role of calreticulin-3-phytaspase interaction in plant innate immunity and senescence can be expected. An intriguing question still remains as to what kind of alteration in posttranslational modification occurs to calreticulin-3 upon phytaspase overproduction in plant cells.

Yet, another potential partner of phytaspase identified in this study, saposin B-type domain-containing protein, also appears to be an interesting candidate. Proteins that harbour such a domain have been reported to localize to the endoplasmic reticulum and plasma membrane. The saposin B domain is capable of associating with membrane lipids and causes distortion and, in some cases, membrane permeabilization [22]. We hypothesize that the interaction with saposin B-containing protein might be related to retrograde trafficking of phytaspase in stressed plant cells, a possibility that is worth addressing in the future.

\section{Materials and Methods}

\subsection{Plant Growth Conditions}

$N$. benthamiana plants were grown at $25^{\circ} \mathrm{C}$ in soil in a controlled environment under a 16/8 h day/night cycle. For transient protein production, Agrobacterium tumefaciens GV3101 cells transformed with the respective plasmid (see below) were mixed with an equal amount of agrobacteria bearing the p19 suppressor of silencing and infiltrated into leaves of six-week-old plants using a blunt syringe.

\subsection{Plasmid Construction}

The ORF of TurboID was amplified by polymerase chain reaction (PCR) using the R4pGWB601_UBQ10p-Turbo-NES-YFP plasmid (a gift from D. Bergmann; Addgene plasmid \#127366) using primers TurboID_Bam_dir and TurboID_His6_z_Sac_rev (Table S1). Six His codons were included in the reverse PCR primer to permit the detection of the recombinant protein. The PCR product (c. $1000 \mathrm{bp}$ long) was cloned between the BamHI and SacI sites of NtPhyt cDNA-carrying pLEX7000 expression plasmid [36] modified to eliminate an additional BamHI site in the polylinker. The resultant pLH-NtPhyt-TurboHis construct contained the NtPhyt-TurboID ORF (the two moieties are separated with a Gly-Gly-Ser spacer) under the control of the CaMV $35 S$ promoter. Insertion of the same PCR product between the BamHI and SacI sites of the pLH_SP_mRFP_His plasmid [15] produced the pLH_SP_Turbo_His construct expressing the SP-TurboID ORF under the control of the $35 \mathrm{~S}$ promoter.

The ORF of N. tabacum calreticulin-3 (c. 1300 bp) was amplified by PCR on total $N$. tabacum cDNA using Phusion Hot Start II High-Fidelity DNA Polymerase (Thermo Scientific, Waltham, MA USA) and CRT_Kpn_Nco_dir and CRT_Bam_rev primers (Table S1), cloned into the pSL1180 vector between the KpnI and BamHI sites, and sequenced to confirm its identity. To construct a plasmid for bacterial production of calreticulin-3 with the signal peptide substituted with the His tag, the calreticulin-3 cDNA fragment was PCR-amplified using CRT_LF_Apa_Nde_dir and CRT_Bam_rev primers. The 1200 bp PCR product was then inserted downstream of and in frame with six His codons between the NdeI and BamHI sites of the pET28a (+) vector (Novagen) to generate the pET_His_LF Calreticulin plasmid encoding His-CRT fusion protein.

\subsection{In Planta Proximity-Dependent Biotinylation Assay}

Leaf discs from $N$. benthamiana plants transiently producing either NtPhyt-Turbo or SP-Turbo protein were prepared 2-3 d post agroinfiltration. Discs were then vacuum infiltrated with $200 \mu \mathrm{M}$ biotin (Sigma) solution in water containing $500 \mu \mathrm{M}$ ATP and $1.25 \mathrm{mM}$ magnesium acetate, and incubated at room temperature for the time periods indicated in the figure legends. 


\subsection{Fractionation of Biotinylated Proteins}

After incubation with biotin, initial steps of leaf protein isolation from different subcellular compartments were performed as described previously [15]. Briefly, apoplastic washes were obtained by low-speed $(2000 \mathrm{~g})$ centrifugation of NtPhyt-TurboID- and SPTurboID-producing leaf discs (typically, $5 \mathrm{~g}$ of leaf material) at $4{ }^{\circ} \mathrm{C}$ for $10 \mathrm{~min}$ and diluted with the ICL buffer (10 mM Tris- $\mathrm{HCl}, \mathrm{pH} 8.8,0.2 \mathrm{M} \mathrm{NaCl}, 30 \mathrm{mM}$ magnesium chloride $\left(\mathrm{MgCl}_{2}\right), 0.2 \mathrm{M}$ sucrose, and $10 \mathrm{mM}$ 2-mercaptoethanol), containing protease inhibitors aprotinin $(2 \mu \mathrm{g} / \mathrm{mL})$, leupeptin $(6 \mu \mathrm{g} / \mathrm{mL})$, chymostatin $(6 \mu \mathrm{g} / \mathrm{mL})$, E64 $(6 \mu \mathrm{g} / \mathrm{mL}), 4$ (2-aminoethyl)benzenesulfonyl fluoride $(25 \mu \mathrm{g} / \mathrm{mL})$, and $2 \mathrm{mM}$ EDTA. The residual leaf material was frozen in liquid nitrogen and disrupted in a Minilys homogenizer (Bertin Instruments, Montigny-le-Bretonneux, France) using $1.4 \mathrm{~mm}$ ceramic beads with two $20 \mathrm{~s}$ bursts. An additional $20 \mathrm{~s}$ burst was performed after suspending the sample in $15 \mathrm{~mL}$ of ICL buffer, and the sample was incubated on ice for 15-30 min. Water-insoluble material was pelleted by centrifugation for $10 \mathrm{~min}$ at $10,000 \mathrm{~g}$ at $4{ }^{\circ} \mathrm{C}$, and the supernatant obtained represented the water-soluble fraction of the intracellular proteins.

Pellets obtained in the previous step contained intracellular proteins that resisted extraction with an aqueous buffer. For their isolation, precipitates were re-suspended in $15 \mathrm{~mL}$ of ICL buffer supplemented with $0.5 \% \mathrm{n}$-dodecyl- $\beta$-D-maltopyranoside (dodecyl maltoside, Anatrace) and protease inhibitors, incubated for $10 \mathrm{~min}$ on ice, and the detergentsolubilized protein fraction was obtained as a supernatant after centrifugation for $10 \mathrm{~min}$ at $10,000 \mathrm{~g}$ at $4{ }^{\circ} \mathrm{C}$.

Both water-soluble and detergent-soluble proteins were further fractionated by ammonium sulphate precipitation. The protein fractions that precipitated within the 0-30, $30-50$, and $50-70 \%$ intervals of $\left(\mathrm{NH}_{4}\right)_{2} \mathrm{SO}_{4}$ saturation were dissolved in $1.5 \mathrm{~mL}$ of $50 \mathrm{mM}$ Tris- $\mathrm{HCl}$ buffer $(\mathrm{pH}$ 7.5) containing protease inhibitors. Biotinylated proteins from these samples were then separated by affinity chromatography using Streptavidin magnetic polymer resin (UBPBio), according to the manufacturer's protocol with modifications. Prior to separation, protein samples were supplied with $1 \%$ sodium dodecyl sulphate (SDS), boiled for $5 \mathrm{~min}$, cooled on ice, and diluted with an equal volume of $50 \mathrm{mM}$ Tris- $\mathrm{HCl}$ buffer (pH 7.5) containing $0.05 \%$ Tween 20 and $1 \mathrm{M} \mathrm{NaCl}$. Magnetic beads $(200 \mu \mathrm{L})$ were washed with $2 \times 1 \mathrm{~mL}$ of $50 \mathrm{mM}$ Tris-HCl buffer (pH 7.5) containing $0.05 \%$ Tween 20 and $0.5 \mathrm{M}$ $\mathrm{NaCl}$ prior to their addition to protein samples and incubated for $4 \mathrm{~h}$ at room temperature with rotation. Beads with bound biotinylated proteins were then separated with a magnet, washed with $3 \times 1 \mathrm{~mL}$ of $50 \mathrm{mM}$ Tris- $\mathrm{HCl}$ buffer ( $\mathrm{pH} 7.5$ ) containing $4 \mathrm{M}$ urea, $0.5 \mathrm{M} \mathrm{NaCl}$, $1 \mathrm{mM}$ dithiothreitol, $0.05 \%$ Tween 20 , and biotinylated proteins were eluted from the beads with $400 \mu \mathrm{L}$ of elution mix ( $2 \%$ SDS, $6 \mathrm{M}$ urea, and $10 \mathrm{mM}$ dithiothreitol) by incubating the samples at $98^{\circ} \mathrm{C}$ for $5 \mathrm{~min}$.

The eluted protein samples were concentrated by overnight precipitation with $80 \%$ acetone. Precipitated proteins were dissolved by pipetting in $50 \mathrm{mM}$ Tris- $\mathrm{HCl}$ buffer ( $\mathrm{pH}$ 8.0) containing 1\% SDS and alkylated by incubation with $10 \mathrm{mM}$ iodoacetamide for $30 \mathrm{~min}$ at room temperature. The reaction was stopped by boiling with the sample buffer, and the protein samples were fractionated by SDS gel electrophoresis. Separated proteins were electrophoretically transferred onto polyvinylidene fluoride (PVDF) membranes and incubated with streptavidin-horseradish peroxidase conjugate (GE Healthcare, for biotinylated proteins) or with HisProbe reagent (Thermo Scientific, for His-tagged proteins). Chemiluminescence detection was performed with ECL Western Lightning Plus reagent (PerkinElmer, Waltham, MA, USA) using the ChemiDoc Imaging System (BioRad Laboratories, Hercules, CA, USA). Data were reproducible over three independent experiments.

\subsection{Activity Assay of NtPhyt}

The proteolytic activity of phytaspase in apoplastic washes, intracellular protein fractions (equivalent to $3 \mathrm{mg}$ of leaf material), and in sub-fractions of the calreticulin-3phytaspase binding assay (1.5 ng NtPhyt in total) was determined using Ac-VEID-AFC 
[AFC, 7-amino-4-(trifluoromethyl) coumarin] fluorogenic peptide substrate (California Peptide), as described in [6]. To perform kinetic measurements of relative fluorescence increase, protein samples were 5-fold diluted with B1 buffer (20 mM MES, $2 \mathrm{mM}$ dithiothreitol, $0.1 \%$ Tween 20, and 5\% glycerol), pH 5.5, containing $0.5 \mathrm{M} \mathrm{NaCl}$ at $28{ }^{\circ} \mathrm{C}$ before activity measurements. The peptide substrate was used at a final concentration of $20 \mu \mathrm{M}$. A FLUOstar OPTIMA reader (BMG Labtech, Ortenberg, Germany) equipped with $405 \mathrm{~nm}$ excitation and $520 \mathrm{~nm}$ emission filters was used to quantitate fluorescence intensities. Data are presented as the means of three independent experiments.

\subsection{MS Analyses}

Matrix-assisted laser desorption ionization-time of flight (MALDI-TOF) MS analysis of tryptic digests was performed on an UltrafleXtreme MALDI-TOF/TOF mass spectrometer (Bruker Daltonics, Bremen, Germany) equipped with an Nd laser by detection of $\mathrm{MH}+$ molecular ions. Pieces of about $2 \mathrm{~mm}^{3}$ of protein-containing gel were de-stained twice with $40 \%$ aqueous acetonitrile solution, dehydrated with $100 \mathrm{~mL}$ of $100 \%$ acetonitrile, and rehydrated with $5 \mathrm{~mL}$ of the digestion solution ( $\mathrm{pH}$ ) containing $15 \mu \mathrm{g} / \mathrm{mL}$ sequencing grade trypsin. Digestion was performed at $37^{\circ} \mathrm{C}$ for $4 \mathrm{~h}$. Proteolysis was stopped by the addition of $10 \mu \mathrm{L}$ of $0.5 \%$ trifluoroacetic acid (TFA). An aliquot of $0.5 \mu \mathrm{L}$ was mixed with $1 \mu \mathrm{L}$ of 2,5 -dihydroxybenzoic acid solution $(40 \mathrm{mg} / \mathrm{mL}$ in $30 \%$ acetonitrile, $0.5 \%$ trifluoroacetic acid (TFA)). The spectra of tryptic digests were recorded in reflector mode, and the accuracy of the monoisotopic mass peak measurement was within 30 ppm. Mass spectra were processed using FlexAnalysis 3.3 software (Bruker Daltonics, Bremen, Germany). Proteins were identified using the home database, which was preloaded with N. benthamiana $\mathrm{NbDE}$ dataset [37] and Mascot combined peptide mass fingerprint + MS/MS search program (Mascot version 2.3.02). The search allowed for one possible missed cleavage, Met oxidation, and Cys-carbamidomethylation. Protein scores greater than 64 were considered to be significant $(p<0.05)$.

\subsection{Calreticulin-3-Nt Phyt In Vitro Binding Assay}

E. coli BL21 (DE3) cells transformed with pET28a-based construct encoding His-CRT3 fusion protein were grown in $20 \mathrm{~mL}$ of LB medium containing kanamycin $(25 \mu \mathrm{g} / \mathrm{mL})$ at $37^{\circ} \mathrm{C}$ with shaking. Exponentially growing cells were shifted to $18{ }^{\circ} \mathrm{C}$, induced with $1 \mathrm{mM}$ isopropyl- $\beta$-D-thiogalactoside, and further incubated overnight at $18{ }^{\circ} \mathrm{C}$ with shaking. Cells (approx. $75 \mathrm{mg}$ ) were pelleted by centrifugation $\left(2000 \times \mathrm{g}\right.$ for $15 \mathrm{~min}$ at $\left.4{ }^{\circ} \mathrm{C}\right)$, resuspended in $5 \mathrm{~mL}$ of sonication buffer ( $50 \mathrm{mM}$ sodium phosphate, $300 \mathrm{mM} \mathrm{NaCl}, \mathrm{pH} 8.0$ ) containing protease inhibitors aprotinin $(2 \mu \mathrm{g} / \mathrm{mL})$, leupeptin $(25 \mu \mathrm{g} / \mathrm{mL})$, chymostatin $(100 \mu \mathrm{g} / \mathrm{mL})$, and phenylmethylsulphonyl fluoride $(10 \mu \mathrm{M})$, sonicated on ice by five $20 \mathrm{~s}$ bursts, and centrifuged at $10,000 \mathrm{~g}$ for $20 \mathrm{~min}$ at $4{ }^{\circ} \mathrm{C}$. The cleared lysate was incubated with Ni-NTA agarose (Qiagen; $200 \mu \mathrm{L}$ of an aqueous suspension per experiment) pre-washed with sonication buffer for $1 \mathrm{~h}$ at $4{ }^{\circ} \mathrm{C}$ with rotation. The resin was then washed with $3 \times 1 \mathrm{~mL}$ of Wash8.0 buffer (50 mM sodium phosphate, $\mathrm{pH} 8.0,1 \mathrm{M} \mathrm{NaCl}, 0.05 \%$ Tween 20, $10 \%$ glycerol), then with $5 \times 0.5 \mathrm{~mL}$ of Wash6.4 buffer $(50 \mathrm{mM}$ sodium phosphate, $\mathrm{pH} 6.4$, $300 \mathrm{mM} \mathrm{NaCl}, 0.05 \%$ Tween 20, 10\% glycerol), and stored on ice. Ni-NTA agarose sample treated in an analogous fashion with a lysate of E. coli BL21 (DE3) cells transformed with an empty pET28a (+) vector served as a control in the NtPhyt binding experiments.

Untagged $N t$ Phyt was affinity-purified from the apoplast of NtPhyt-overproducing $N$. benthamiana leaves using the reversible biotinylated peptide aldehyde inhibitor BioTATD-CHO (Bachem) as described in [6]. To assess CRT3-NtPhyt binding, an aliquot $(5 \mu \mathrm{L})$ of Ni-NTA agarose with immobilized His-CRT3 (approx. $0.5 \mu \mathrm{g}$ protein per binding experiment), or an equivalent amount of the control resin, was washed with $4 \times 50 \mu \mathrm{L}$ of $20 \mathrm{mM}$ MES buffer ( $\mathrm{pH}$ 6.5) and incubated with NtPhyt (15 ng) in a total volume of $30 \mu \mathrm{L}$ of the same buffer for $3 \mathrm{~h}$ on ice. After centrifugation at 12,000 $\mathrm{g}$ for $15 \mathrm{~s}$, unbound $\mathrm{NtPhyt}$ contained in the flow-through and in $2 \times 30 \mu \mathrm{L}$ washes with $20 \mathrm{mM}$ MES buffer ( $\mathrm{pH}$ 6.5) was combined, and the resin-bound proteins were eluted by $10 \mathrm{~min}$ incubations on ice with 
$3 \times 30 \mu \mathrm{L}$ of B1 buffer, $\mathrm{pH} 5.5$, containing $50 \mathrm{mM} \mathrm{NaCl}$ and $100 \mathrm{mM}$ EDTA. Aliquots (10\%) of the combined eluates and unbound proteins were used to determine NtPhyt activity. Aliquots of eluates were analysed by SDS-gel electrophoresis with Coomassie staining to evaluate the amount of His-CRT3 in the samples. Experiments with NtPhyt binding were performed in triplicate. Data are presented as means from these experiments. Statistical analysis was performed using GraphPad Prism 8.0.1 software (GraphPad Software, San Diego, CA, USA). Statistical significance was analysed using the unpaired t-test. The level of significance is indicated by asterisks in Figure 6.

Supplementary Materials: The following are available online at https:/ / www.mdpi.com/article/10 $.3390 /$ ijms222313123/s1

Author Contributions: Conceptualization, A.B.V. and N.V.C.; Supervision, A.B.V. and N.V.C.; Investigation, A.D.T., M.V.S., R.A.G. and N.V.C.; Formal Analysis, A.D.T., M.V.S., R.A.G., N.V.C. and A.B.V.; Writing-Original Draft Preparation, A.B.V.; Writing-Review and Editing, A.D.T., M.V.S. and N.V.C. All authors have read and agreed to the published version of the manuscript.

Funding: This work was funded by the Russian Science Foundation, grant number 19-14-00010.

Institutional Review Board Statement: Not applicable.

Informed Consent Statement: Not applicable.

Data Availability Statement: Exclude.

Acknowledgments: MALDI MS facility became available to us in the framework of the Moscow State University Development Program PNG 5.13.

Conflicts of Interest: The authors declare no conflict of interest.

\section{References}

1. Schaller, A.; Stintzi, A.; Rivas, S.; Serrano, I.; Chichkova, N.V.; Vartapetian, A.B.; Martínez, D.; Guiamét, J.J.; Sueldo, D.J.; van der Hoorn, R.A.L.; et al. From structure to function-A family portrait of plant subtilases. New Phytol. 2018, 218, 901-915. [CrossRef] [PubMed]

2. Rautengarten, C.; Steinhauser, D.; Büssis, D.; Stintzi, A.; Schaller, A.; Kopka, J.; Altmann, T. Inferring hypotheses on functional relationships of genes: Analysis of the Arabidopsis thaliana subtilase gene family. PLoS Comput. Biol. 2005, 1, e40. [CrossRef]

3. Martinez, D.E.; Borniego, M.L.; Battchikova, N.; Aro, E.M.; Tyystjärvi, E.; Guiamét, J.J. SASP, a senescence-associated subtilisin protease, is involved in reproductive development and determination of silique number in Arabidopsis. J. Exp. Bot. 2015, 66, 161-174. [CrossRef] [PubMed]

4. $\quad$ Roberts, I.N.; Passeron, S.; Barneix, A.J. The two main endoproteases present in dark-induced senescent wheat leaves are distinct subtilisin-like proteases. Planta 2006, 224, 1437-1447. [CrossRef]

5. Roberts, I.N.; Caputo, C.; Kade, M.; Criado, M.V.; Barneix, A.J. Subtilisin-like serine proteases involved in N remobilization during grain filling in wheat. Acta Physiol. Plant. 2011, 33, 1997-2001. [CrossRef]

6. Chichkova, N.V.; Shaw, J.; Galiullina, R.A.; Drury, G.E.; Tuzhikov, A.I.; Kim, S.H.; Kalkum, M.; Hong, T.B.; Gorshkova, E.N.; Torrance, L.; et al. Phytaspase, a relocalisable cell death promoting plant protease with caspase specificity. EMBO J. 2010, 29, 1149-1461. [CrossRef] [PubMed]

7. Galiullina, R.A.; Kasperkiewicz, P.; Chichkova, N.V.; Szalek, A.; Serebryakova, M.V.; Poreba, M.; Drag, M.; Vartapetian, A.B. Substrate specificity and possible heterologous targets of phytaspase, a plant cell death protease. J. Biol. Chem. 2015, 290, 24806-24815. [CrossRef] [PubMed]

8. Reichardt, S.; Repper, D.; Tuzhikov, A.I.; Galiullina, R.A.; Planas-Marquès, M.; Chichkova, N.V.; Vartapetian, A.B.; Stintzi, A.; Schaller, A. The tomato subtilase family includes several cell death-related proteinases with caspase specificity. Sci. Rep. 2018, 8 , 10531. [CrossRef]

9. Chichkova, N.V.; Galiullina, R.A.; Mochalova, L.V.; Trusova, S.V.; Sobri, Z.M.; Gallois, P.; Vartapetian, A.B. Arabidopsis thaliana phytaspase: Identification and peculiar properties. Funct. Plant. Biol. 2018, 45, 171-179. [CrossRef]

10. Vartapetian, A.B.; Tuzhikov, A.I.; Chichkova, N.V.; Taliansky, M.; Wolpert, T.J. A plant alternative to animal caspases: Subtilisinlike proteases. Cell Death Differ. 2011, 18, 1289-1297. [CrossRef]

11. Chichkova, N.V.; Kim, S.H.; Titova, E.S.; Kalkum, M.; Morozov, V.S.; Rubtsov, Y.P.; Kalinina, N.O.; Taliansky, M.E.; Vartapetian, A.B. A plant caspase-like protease activated during the hypersensitive response. Plant Cell. 2004, 16, 157-171. [CrossRef] [PubMed]

12. Chichkova, N.V.; Tuzhikov, A.I.; Taliansky, M.; Vartapetian, A.B. Plant phytaspases and animal caspases: Structurally unrelated death proteases with a common role and specificity. Physiol. Plant. 2012, 145, 77-84. [CrossRef] 
13. Fomicheva, A.S.; Tuzhikov, A.I.; Beloshistov, R.E.; Trusova, S.V.; Galiullina, R.A.; Mochalova, L.V.; Chichkova, N.V.; Vartapetian, A.B. Programmed cell death in plants. Biochemistry 2012, 77, 1452-1464. [CrossRef] [PubMed]

14. Trusova, S.V.; Golyshev, S.A.; Chichkova, N.V.; Vartapetian, A.B. Sometimes they come back: Endocytosis provides localization dynamics of a subtilase in cells committed to cell death. J. Exp. Bot. 2019, 70, 2003-2007. [CrossRef] [PubMed]

15. Trusova, S.V.; Teplova, A.D.; Golyshev, S.A.; Galiullina, R.A.; Morozova, E.A.; Chichkova, N.V.; Vartapetian, A.B. Clathrinmediated endocytosis delivers proteolytically active phytaspases into plant cells. Front. Plant Sci. 2019, 10, 873. [CrossRef] [PubMed]

16. Roux, K.J.; Kim, D.I.; Raida, M.; Burke, B. A promiscuous biotin ligase fusion protein identifies proximal and interacting proteins in mammalian cells. J. Cell Biol. 2012, 196, 801-810. [CrossRef] [PubMed]

17. Christensen, A.; Svensson, K.; Thelin, L.; Zhang, W.; Tintor, N.; Prins, D.; Funke, N.; Michalak, M.; Schulze-Lefert, P.; Saijo, Y.; et al. Higher plant calreticulins have acquired specialized functions in Arabidopsis. PLoS ONE 2010, 5, e11342. [CrossRef]

18. Branon, T.C.; Bosch, J.A.; Sanchez, A.D.; Udeshi, N.D.; Svinkina, T.; Carr, S.A.; Feldman, J.L.; Perrimon, N.; Ting, A.Y. Efficient proximity labeling in living cells and organisms with TurboID. Nat. Biotechnol. 2018, 36, 880-887. [CrossRef] [PubMed]

19. Mair, A.; Xu, S.L.; Branon, T.C.; Ting, A.Y.; Bergmann, D.C. Proximity labeling of protein complexes and cell-type-specific organellar proteomes in Arabidopsis enabled by TurboID. Elife 2019, 8, e47864. [CrossRef] [PubMed]

20. Zhang, Y.; Song, G.; Lal, N.K.; Nagalakshmi, U.; Li, Y.; Zheng, W.; Huang, P.J.; Branon, T.C.; Ting, A.Y.; Walley, J.W.; et al. TurboID-based proximity labeling reveals that UBR7 is a regulator of N NLR immune receptor-mediated immunity. Nat. Commun. 2019, 10, 3252. [CrossRef] [PubMed]

21. Arora, D.; Abel, N.B.; Liu, C.; Van Damme, P.; Yperman, K.; Eeckhout, D.; Vu, L.D.; Wang, J.; Tornkvist, A.; Impens, F.; et al. Establishment of proximity-dependent biotinylation approaches in different plant model systems. Plant Cell 2020, 32, 3388-3407. [CrossRef]

22. Bruhn, H. A short guided tour through functional and structural features of saposin-like proteins. Biochem. J. 2005, 389, 249-257. [CrossRef] [PubMed]

23. Denecke, J.; Carlsson, L.E.; Vidal, S.; Höglund, A.S.; Ek, B.; van Zeijl, M.J.; Sinjorgo, K.M.; Palva, E.T. The tobacco homolog of mammalian calreticulin is present in protein complexes in vivo. Plant Cell 1995, 7, 391-406.

24. Lin, Q.; Zhou, Z.; Luo, W.; Fang, M.; Li, M.; Li, H. Screening of proximal and interacting proteins in rice protoplasts by proximity-dependent biotinylation. Front. Plant Sci. 2017, 8, 749. [CrossRef] [PubMed]

25. Khan, M.; Youn, J.Y.; Gingras, A.C.; Subramaniam, R.; Desveaux, D. In planta proximity dependent biotin identification (BioID). Sci. Rep. 2018, 8, 9212. [CrossRef] [PubMed]

26. Arosa, F.A.; de Jesus, O.; Porto, G.; Carmo, A.M.; de Sousa, M. Calreticulin is expressed on the cell surface of activated human peripheral blood T lymphocytes in association with major histocompatibility complex class I molecules. J. Biol. Chem. 1999, 274, 16917-16922. [CrossRef] [PubMed]

27. Holaska, J.M.; Black, B.E.; Love, D.C.; Hanover, J.A.; Leszyk, J.; Paschal, B.M. Calreticulin Is a receptor for nuclear export. J. Cell Biol. 2001, 152, 127-140. [CrossRef] [PubMed]

28. Gardai, S.J.; McPhillips, K.A.; Frasch, S.C.; Janssen, W.J.; Starefeldt, A.; Murphy-Ullrich, J.E.; Bratton, D.L.; Oldenborg, P.A.; Michalak, M.; Henson, P.M. Cell-surface calreticulin initiates clearance of viable or apoptotic cells through trans-activation of LRP on the phagocyte. Cell 2005, 123, 321-334. [CrossRef]

29. Gold, L.I.; Eggleton, P.; Sweetwyne, M.T.; Van Duyn, L.B.; Greives, M.R.; Naylor, S.M.; Michalak, M.; Murphy-Ullrich, J.E. Calreticulin: Non-endoplasmic reticulum functions in physiology and disease. FASEB J. 2010, 24, 665-683. [CrossRef] [PubMed]

30. Persson, S.; Rosenquist, M.; Svensson, K.; Galvão, R.; Boss, W.F.; Sommarin, M. Phylogenetic analyses and expression studies reveal two distinct groups of calreticulin isoforms in higher plants. Plant Physiol. 2003, 133, 1385-1396. [CrossRef]

31. Li, J.; Zhao-Hui, C.; Batoux, M.; Nekrasov, V.; Roux, M.; Chinchilla, D.; Zipfel, C.; Jones, J.D. Specific ER quality control components required for biogenesis of the plant innate immune receptor EFR. Proc. Natl. Acad. Sci. USA 2009, 106, 15973-15978. [CrossRef]

32. Jin, H.; Hong, Z.; Su, W.; Li, J. A plant-specific calreticulin is a key retention factor for a defective brassinosteroid receptor in the endoplasmic reticulum. Proc. Natl. Acad. Sci. USA 2009, 106, 13612-13617. [CrossRef] [PubMed]

33. Liebrand, T.W.; Smit, P.; Abd-El-Haliem, A.; de Jonge, R.; Cordewener, J.H.; America, A.H.; Sklenar, J.; Jones, A.M.; Robatzek, S.; Thomma, B.P.; et al. Endoplasmic reticulum-quality control chaperones facilitate the biogenesis of Cf receptor-like proteins involved in pathogen resistance of tomato. Plant Physiol. 2012, 159, 1819-1833. [CrossRef] [PubMed]

34. Qiu, Y.; Xi, J.; Du, L.; Poovaiah, B.W. The function of calreticulin in plant immunity: New discoveries for an old protein. Plant Signal Behav. 2012, 7, 907-910. [CrossRef]

35. Joshi, R.; Paul, M.; Kumar, A.; Pandey, D. Role of calreticulin in biotic and abiotic stress signalling and tolerance mechanisms in plants. Gene 2019, 714, 144004. [CrossRef] [PubMed]

36. Beloshistov, R.E.; Dreizler, K.; Galiullina, R.A.; Tuzhikov, A.I.; Serebryakova, M.V.; Reichardt, S.; Shaw, J.; Taliansky, M.E.; Pfannstiel, J.; Chichkova, N.V.; et al. Phytaspase-mediated precursor processing and maturation of the wound hormone systemin. New Phytol. 2018, 218, 1167-1178. [CrossRef] [PubMed]

37. Kourelis, J.; Kaschani, F.; Grosse-Holz, F.M.; Homma, F.; Kaiser, M.; van der Hoorn, R.A.L. A homology-guided, genome-based proteome for improved proteomics in the alloploid Nicotiana benthamiana. BMC Genom. 2019, 20, 722. [CrossRef] [PubMed] 